\title{
Online Scheduling with Delivery Time on a Bounded Parallel Batch Machine with Limited Restart
}

\author{
Hailing Liu, ${ }^{1,2}$ Long Wan, ${ }^{3}$ Zhigang Yan, ${ }^{4}$ and Jinjiang Yuan' \\ ${ }^{1}$ School of Mathematics and Statistics, Zhengzhou University, Zhengzhou, Henan 450001, China \\ ${ }^{2}$ College of Science, Henan Institute of Engineering, Zhengzhou, Henan 451191, China \\ ${ }^{3}$ School of Information Technology, Jiangxi University of Finance and Economics, Nanchang 330013, China \\ ${ }^{4}$ Accounting School, Jiangxi University of Finance and Economics, Nanchang 330013, China \\ Correspondence should be addressed to Long Wan; cocu3328@163.com
}

Received 23 August 2014; Accepted 28 September 2014

Academic Editor: Yunqiang Yin

Copyright ( 2015 Hailing Liu et al. This is an open access article distributed under the Creative Commons Attribution License, which permits unrestricted use, distribution, and reproduction in any medium, provided the original work is properly cited.

\begin{abstract}
We consider the online (over time) scheduling of equal length jobs on a bounded parallel batch machine with batch capacity $b$ to minimize the time by which all jobs have been delivered with limited restart. Here, "restart" means that a running batch may be interrupted, losing all the work done on it, and jobs in the interrupted batch are then released and become independently unscheduled jobs, called restarted jobs. "Limited restart" means that a running batch which contains some restarted jobs cannot be restarted again. When $b=2$, we propose a best possible online algorithm $H(b=2)$ with a competitive ratio of $1+\alpha$, where $\alpha$ is the positive solution of $2 \alpha(1+\alpha)=1$. When $b \geq 3$, we present a best possible online algorithm $H(b \geq 3)$ with a competitive ratio of $1+\beta$, where $\beta$ is the positive solution of $\beta(1+\beta)^{2}=1$.
\end{abstract}

\section{Introduction}

In this paper, we consider the online scheduling of equal length jobs on a bounded parallel batch machine with batch capacity $b$ to minimize the time by which all jobs have been delivered with limited restart. Here, a bounded parallel batch machine is a machine which can process up to $b$ jobs simultaneously as a batch, where $b$ is the batch capacity. The processing time of a batch is the longest processing time of jobs in the batch. Jobs in a batch have the same starting time and the same completion time. According to the characteristic of batch capacity $b$, there are the bounded model and the unbounded model. The bounded model means that $b$ is finite; that is, $b<\infty$. The unbounded model means that $b$ can be as large as possible; that is, $b=\infty$. In this paper, we study the bounded model, that is, bounded parallel batch machine.

In this paper, online means that each job becomes available at its arrival time which is unknown beforehand and the characteristics of the job are unknown until it arrives.
Each job $J_{j}$ has an arrival time $r_{j}$, a processing time $p_{j}$, and a delivery time $q_{j}$. In our research, we assume that all jobs have equal processing times. By scaling, we may assume that each job has a processing time 1. Each job needs to be processed on the bounded parallel batch machine and then delivered to the destination by a delivery vehicle. Here we assume that there are sufficiently many delivery vehicles, which means that a job can be transported as soon as it is completed on the bounded parallel batch machine. Let $C_{j}$ be the completion time of $J_{j}$ on the bounded parallel batch machine and let $L_{j}$ be the time by which $J_{j}$ has been delivered. Then $L_{j}=C_{j}+q_{j}$. Let $L_{\text {max }}=\max _{j}\left\{L_{j}: L_{j}=C_{j}+q_{j}\right\}$. Our objective is to minimize $L_{\max }$, that is, the time by which all jobs have been delivered.

The quality of an online algorithm is measured by the competitive ratio. An online algorithm is called $\rho$-competitive if, for any input instance, it produces a schedule with an objective value not worse than $\rho$ times the value of an optimal offline schedule. 
Restart (see Hoogeveen et al. [1]) means that a running task may be interrupted, losing all the work done on it. The jobs in the interrupted task, which are called restarted jobs, are then released and become independently unscheduled jobs which can be scheduled anew later. Using restarts means that we have a chance to change our original mind to get a better schedule according to the newly arrived jobs and so can efficiently improve the quality of some online algorithms. For example, for the problem of minimizing the time by which all jobs have been delivered on a single machine without restarts, the best possible online algorithm has a competitive ratio of $(\sqrt{5}+1) / 2$. While using restarts, a best possible online algorithm with a competitive ratio of $3 / 2$ was given in Akker et al. [2]. Other researches with restarts can see Epstein and Stee [3], Stee and Poutré [4], and Yuan et al. [5].

Limited restart is first introduced by Fu et al. [6]. Limited restart means that once a batch contains some restarted jobs, we cannot restart the batch again. Limited restart implies that a job can be restarted at most once. Limited restart is of practical value. In fact, too many restarts of a job may cause the waste of cost and improve the possibility of a spoiled product.

Parallel batching scheduling is motivated by burn in operations in semiconductor manufacture (see $[7,8])$. For online scheduling on a single unbounded parallel batch machine to minimize makespan, that is, the time by which all jobs are completed on the parallel batch machine, Deng et al. [9] and Zhang et al. [10] independently gave the same online algorithm with the competitive ratio of $(\sqrt{5}+1) / 2$ and proved it is best possible. For online scheduling on a single bounded parallel batch machine to minimize makespan, Poon and Yu [11] proved that any FBLPT-based algorithm is 2-competitive. Particularly for batch capacity $b=2$, they presented an online algorithm with the competitive ratio of $7 / 4$. For online scheduling on $m$ unbounded parallel batch machines to minimize makespan, Liu et al. [12] and Tian et al. [13] independently presented two different but best possible online algorithms with a competitive ratio of $1+\alpha_{m}$, where $\alpha_{m}$ is the positive solution of the equation $a_{m}^{2}+m \alpha_{m}-1=$ 0 . For online scheduling on a single parallel batch machine to minimize $L_{\max }$, that is, the time by which all jobs have been delivered, Tian et al. [14] gave a 2-competitive online algorithm for the unbounded model and a 3-competitive online algorithm for the bounded model. Moreover, when each job has the same processing time, they provided two $(\sqrt{5}+1) / 2$-competitive optimal online algorithms for the two models, respectively. Yuan et al. [15] gave a best possible online algorithm with a competitive ratio of $(\sqrt{5}+1) / 2$ for the two models of $q_{j} \leq p_{j}$ and $\left[p_{i}>p_{j}\right.$ implying $q_{i} \geq q_{j}$ ]. Fang et al. [16] studied the restricted model that all jobs have their processing times in $[p,((1+\sqrt{5}) / 2) p]$ and provided a class of optimal online algorithms with a competitive ratio of $(1+\sqrt{5}) / 2$. Tian et al. [17] presented a $(2 \sqrt{2}-1)$-competitive online algorithm for the unbounded model. Liu and $\mathrm{Lu}$ [18] studied online scheduling on $m$ unbounded parallel batch machines to minimize $L_{\text {max }}$. For the restricted model of $\left[p_{i}>p_{j}\right.$ implying $q_{i} \geq q_{j}$ ], they gave a best possible online algorithm with competitive ratio of $1+\alpha_{m}$, where $\alpha_{m}$ is the positive solution of $\alpha_{m}^{2}+m \alpha_{m}=1$. For the general case, they gave an online algorithm with competitive ratio of $1+2 /\lfloor m\rfloor$.

For the problem of minimizing makespan on an unbounded parallel batch machine with restarts, Fu et al. [19] showed that any online algorithm cannot have a competitive ratio of less than $(5-\sqrt{5}) / 2$ and Yuan et al. [5] gave a best possible online algorithm matching the lower bound. For the corresponding problem using limited restart, Fu et al. [6] gave an online algorithm with a competitive ratio of $3 / 2$ and proved that it is best possible. For the problem of minimizing makespan on two unbounded parallel batch machines with limited restart, Fu et al. [20] provided a best possible online algorithm which is $(\sqrt{3}+1) / 2$-competitive under the secondrestart assumption. Recently, Liu and Yuan [21] presented best possible online algorithms for minimizing makespan of equal length jobs on a bounded parallel batch machine with limited restart or restart.

Let $J$ be a job set. Then $|J|$ is used to denote the number of jobs in $J$. Let $q(J)$ denote the largest delivery time of jobs in $J$ and let $r(J)$ denote the latest arrival time of jobs whose delivery times are $q(J)$ in $J$. For a batch $B$ in some schedule, $S(B)$ is starting time of $B$ on the parallel batch machine. Batch $B$ is called full if $|B|=b$ and nonfull if $|B|<b$. In an online algorithm $H$ which generates a schedule consisting of $n$ processing batches, let $B_{i}$ denote the $i$ th processing batch such that $S\left(B_{1}\right)<S\left(B_{2}\right)<\cdots<S\left(B_{n}\right)$. For simplicity, we write $S\left(B_{i}\right)$ as $S_{i}$. Let $U(t)$ be the set of the available unscheduled jobs at time $t$ in $H$.

If, in an online algorithm $H, B_{k}$ is being processed at the current time $t$, then the following subprocedure which is called Restart $(k, t)$ may be used.

Restart $(k, t)$. At time $t$, interrupt $B_{k}$ and select $\min \left\{b, \mid B_{k} \cup\right.$ $U(t) \mid\}$ jobs in $B_{k} \cup U(t)$ such that each job selected has a delivery time not less than the largest delivery time of jobs not selected in $B_{k} \cup U(t)$ as the batch $B_{k+1}$ starting at time $t$. Then reset $k$ to be $k+1$.

This paper studies the online scheduling of equal length jobs on a bounded parallel batch machine with batch capacity $b$ to minimize $L_{\max }$ with limited restart. Here $L_{\max }$ means the time by which all jobs have been delivered. This problem can be denoted by $1 \quad$ online, $r_{j}, p_{j}=1$, P-batch, $b<$ $\infty$, L-restart $\mid L_{\max }$. For simplicity, when $b=2$, the problem is shortly written as $\mathrm{P}$-batch $(b=2)$. When $b \geq 3$, the problem is shortly written as P-batch $(b \geq 3)$.

Let $\alpha$ be the positive solution of $2 \alpha(1+\alpha)=1$ and let $\beta$ be the positive solution of $\beta(1+\beta)^{2}=1$.

This paper is organized as follows. In Section 2, we give a best possible online algorithm with a competitive ratio of $1+\alpha$ for problem P-batch $(b=2)$. In Section 3 , we give a best possible online algorithm with a competitive ratio of $1+\beta$ for problem P-batch $(b \geq 3)$.

\section{Problem P-Batch $(b=2)$}

For a job instance $I$, let $L_{\text {on }}=L_{\text {on }}(I)$ and $L_{\text {opt }}=L_{\text {opt }}(I)$ denote, respectively, the values of $L_{\max }$ given by an online algorithm and an offline optimal algorithm. 
Theorem 1. Any online algorithm for problem P-batch $(b=2)$ cannot have a competitive ratio of less than $1+\alpha$.

Proof. Let $\epsilon$ be a sufficiently small positive number. For any online algorithm $H$, we consider the following instance. At time 0 , the first job $J_{1}$ with $q_{1}=0$ arrives and no jobs arrive before the starting time of $J_{1}$.

If $S_{1} \geq \alpha$, no jobs arrive later. We have $L_{\text {on }}=S_{1}+1$ and $L_{\text {opt }}=1$. Hence, $L_{\text {on }} / L_{\text {opt }}=\left(S_{1}+1\right) / 1 \geq 1+\alpha$.

If $S_{1}<\alpha$, then, at time $S_{1}+\alpha$, the second job $J_{2}$ with $q_{2}=0$ arrives and no jobs arrive before the starting time of $J_{2}$. We distinguish the following two cases.

Case $1\left(S_{2} \geq S_{1}+1\right)$. Then no jobs arrive later. Then $L_{\text {on }} \geq S_{1}+2$ and $L_{\text {opt }}=S_{1}+\alpha+1$. Thus $L_{\text {on }} / L_{\text {opt }} \geq\left(S_{1}+2\right) /\left(S_{1}+\alpha+1\right) \geq$ $(\alpha+2) /(2 \alpha+1)=1+\alpha$.

Case $2\left(S_{2}<S_{1}+1\right)$. Then $B_{2}$ interrupts $B_{1}$.

If $B_{2}$ is a batch containing only one job $J_{2}$, no jobs arrive later. We have $L_{\text {on }} \geq S_{2}+2 \geq S_{1}+\alpha+2$ and $L_{\text {opt }}=S_{1}+\alpha+1$. Hence, $L_{\text {on }} / L_{\text {opt }} \geq\left(S_{1}+\alpha+2\right) /\left(S_{1}+\alpha+1\right) \geq 1+1 /(2 \alpha+1)>$ $1+\alpha$.

If $B_{2}$ is a batch consisting of $\left\{J_{1}, J_{2}\right\}$ and $S_{2} \leq 2 \alpha$, then job $J_{3}$ with $q_{3}=1$ arrives at time $S_{2}+\epsilon$ and no jobs arrive later. As $B_{2}$ cannot be restarted, we have $L_{\text {on }} \geq S_{2}+2+q_{3}=S_{2}+2+1$. In the offline setting, we can schedule $J_{3}$ starting at time $S_{2}+\epsilon$ and $\left\{J_{1}, J_{2}\right\}$ as a single batch starting at time $S_{2}+1+\epsilon$. So $L_{\text {opt }} \leq S_{2}+2+\epsilon$. Then when $\epsilon \rightarrow 0$, we have $L_{\text {on }} / L_{\text {opt }} \geq$ $\left(S_{2}+3\right) /\left(S_{2}+2+\epsilon\right) \rightarrow\left(S_{2}+3\right) /\left(S_{2}+2\right) \geq 1+1 /(2 \alpha+2)=$ $1+\alpha$.

If $B_{2}$ is a batch consisting of $\left\{J_{1}, J_{2}\right\}$ and $S_{2}>2 \alpha$, then job $J_{3}$ with $q_{3}=0$ arrives at time $S_{2}+\epsilon$ and no jobs arrive later. As $B_{2}$ cannot be restarted, we have $L_{\text {on }} \geq$ $S_{2}+2$. In the offline setting, we can schedule $J_{1}$ starting at time 0 and $\left\{J_{2}, J_{3}\right\}$ as a single batch starting at time $\max \left\{1, S_{2}+\epsilon\right\}$. So $L_{\text {opt }} \leq \max \left\{2, S_{2}+1+\epsilon\right\}$. Then when $\epsilon \rightarrow 0$, we have $L_{\text {on }} / L_{\text {opt }} \geq\left(S_{2}+2\right) / \max \left\{2, S_{2}+1+\right.$ $\epsilon\} \rightarrow\left(S_{2}+2\right) / \max \left\{2, S_{2}+1\right\}=\min \left\{\left(S_{2}+2\right) / 2,1+\right.$ $\left.1 /\left(S_{2}+1\right)\right\} \geq \min \{1+\alpha, 1+1 /(\alpha+2)\}=1+$ $\alpha$.

The theorem follows.

Algorithm $H(b=2)$. Initially set $k=0$. At the current time instant $t$, do the following.

$S(1)$ : if $k=0, U(t) \neq \emptyset$, and $t \geq \alpha$, then select $\min \{2,|U(t)|\}$ jobs in $U(t)$ such that each job selected has a delivery time not less than the largest delivery time of jobs not selected in $U(t)$ as the batch $B_{k+1}$ starting at time $t$. Then reset $k$ to be $k+1$.

$S(2)$ : if $k \geq 1$, the machine is idle, and $U(t) \neq \emptyset$, then select $\min \{2,|U(t)|\}$ jobs in $U(t)$ such that each job selected has a delivery time not less than the largest delivery time of jobs not selected in $U(t)$ as the batch $B_{k+1}$ starting at time $t$. Then reset $k$ to be $k+1$.

$\mathrm{S}(3)$ : if the machine is occupied by $B_{k}, k \in\{1,2\}, U(t) \neq \emptyset$, and each batch starting earlier than $S_{k}$ is not interrupted, do the following.

$\mathrm{S}(3.1)$ : if $B_{k}$ is nonfull and $t=S_{k}+\alpha$, then do Restart $(k, t)$.
$\mathrm{S}(3.2)$ : if $k=1, B_{k}$ is full, $t=S_{k}+\alpha$, and some job in $U(t)$ has a delivery time larger than the delivery time of some job in $B_{k}$, then do Restart $(k, t)$.

$S(4)$ : otherwise, do nothing but wait.

From algorithm $H(b=2)$, we have the following observation.

Observation 1. (1) For each batch $B_{i}$ in algorithm $H(b=2)$, we have $S_{i} \geq \alpha$.

(2) All jobs arrive at or after $S_{1}-\alpha$. And if $S_{1}>\alpha$, then all jobs arrive at or after $S_{1}$.

(3) If $B_{j}$ interrupts $B_{j-1}$, then $S_{j}=S_{j-1}+\alpha$.

(4) If $k \geq 3$, then $S_{k} \geq 2 \alpha+1$.

Theorem 2. For problem P-batch $(b=2), H(b=2)$ is a best possible online algorithm with a competitive ratio of $1+\alpha$.

Proof. Suppose to the contrary that $I$ is an instance so that $L_{\text {on }} / L_{\text {opt }}>1+\alpha$. Let $\sigma$ be the schedule generated by running algorithm $H(b=2)$, for instance, $I$. For simplicity, in the following we always write $q\left(B_{i}\right)$ as $q_{i}$ and write $r\left(B_{i}\right)$ as $r_{i}$ without causing confusion. By the definitions of $q\left(B_{i}\right)$ and $r\left(B_{i}\right)$, we know that $q_{i}$ is the largest delivery time of jobs in $B_{i}$ and $r_{i}$ is the latest arrival time of jobs whose delivery times are $q_{i}$ in $B_{i}$. Let $B_{n}$ be the earliest starting batch in $\sigma$ which assumes $L_{\text {on }}$ and is not interrupted. Then $L_{\text {on }}=S_{n}+1+q_{n}$. Let $\pi$ be an offline optimal schedule, for instance, $I$. As $S_{1} \leq$ $\max \left\{r_{1}, \alpha\right\}$, then the assumption that $L_{\text {on }} / L_{\text {opt }}>1+\alpha$ implies $n \geq 2$.

Let $C_{\text {on }}=S_{n}+1$; that is, $C_{\text {on }}$ is the completion time of $B_{n}$ on the parallel batch machine. Let $t$ be the minimum time instant such that, in $\sigma$, there are no idle times between $t$ and $C_{\text {on }}$ and each batch processed in the time interval $\left[t, C_{\text {on }}\right]$ is not interrupted. Suppose that there are $h$ batches $B_{n-h+1}, \ldots, B_{n}$ processed in the time interval $\left[t, C_{\text {on }}\right]$ in $\sigma$. Then $C_{\text {on }}=t+h$. Set $n^{\prime}=n-h+1$. So $S_{n^{\prime}}=t$.

Claim 1 ( $B_{n}$ does not interrupt $B_{n-1}$ and $h \geq 2$ ). Otherwise, $B_{n}$ interrupts $B_{n-1}$. Then $L_{\text {on }}=S_{n}+1+q_{n}=S_{n-1}+\alpha+1+q_{n}$. By algorithm $H(b=2)$, we have $n=2,3$. Let $J^{\prime}$ be the job with the largest delivery time among jobs arriving in the interval $\left(S_{n-1}, S_{n}\right]$, let $q^{\prime}$ be the delivery time of $J^{\prime}$, and let $r^{\prime}$ be the arrival time of $J^{\prime}$. Then $q_{n}=\max \left\{q_{n-1}, q^{\prime}\right\}$. If $q^{\prime} \geq q_{n-1}$, then $q_{n}=q^{\prime}, L_{\text {opt }} \geq r^{\prime}+1+q^{\prime}>S_{n-1}+1+q^{\prime}, L_{\text {on }}=S_{n-1}+\alpha+1+q^{\prime}$, and, accordingly, $L_{\text {on }} / L_{\text {opt }}<1+\alpha$, a contradiction. If there exists only one batch in the offline optimal schedule $\pi$, then $L_{\text {opt }} \geq r^{\prime}+1+q_{n}>S_{n-1}+1+q_{n}$, and accordingly, $L_{\text {on }} / L_{\text {opt }} \leq$ $\left(S_{n-1}+\alpha+1+q_{n}\right) /\left(S_{n-1}+1+q_{n}\right)<1+\alpha$, a contradiction. So in the following we assume that $q^{\prime}<q_{n-1}$, that is, $q_{n}=q_{n-1}$, and assume that there exist at least two batches in the offline optimal schedule $\pi$. Then $L_{\text {on }}=S_{n-1}+\alpha+1+q_{n-1}$ and $L_{\text {opt }} \geq 2$.

If $n=2$, then $L_{\text {on }}=S_{1}+\alpha+1+q_{1}$. By Observation 1, we have $L_{\text {opt }} \geq S_{1}-\alpha+1+q_{1}$. Noticing that $L_{\text {opt }} \geq 2$, we have $L_{\text {on }} / L_{\text {opt }} \leq 1+\alpha$, a contradiction.

If $n=3$, then $L_{\text {on }}=S_{2}+\alpha+1+q_{2}$. By algorithm $H(b=2)$, we know that $B_{2}$ does not interrupt $B_{1}$; that is, $S_{2} \geq S_{1}+1$. If $S_{2}>S_{1}+1$, then all jobs in $B_{2}$ arrive at or after $S_{2}$, and so $L_{\text {opt }} \geq S_{2}+1+q_{2}$. Thus, $L_{\text {on }} / L_{\text {opt }}<1+\alpha$, a contradiction. If 
$S_{2}=S_{1}+1$, then $L_{\text {on }}=S_{1}+\alpha+2+q_{2}$. If, in the offline optimal schedule $\pi, J_{2}$ is scheduled after the first starting batch, then $L_{\text {opt }} \geq S_{1}-\alpha+2+q_{2}$. Thus, $L_{\text {on }} / L_{\text {opt }} \leq\left(S_{1}+\alpha+2+q_{2}\right) /\left(S_{1}-\right.$ $\left.\alpha+2+q_{2}\right) \leq 1+\alpha$, a contradiction. If, in the offline optimal schedule $\pi, J_{2}$ is scheduled in the first starting batch and $r_{2} \geq$ $S_{1}+\alpha$, considering there exist at least two batches in the offline optimal schedule $\pi$, then $L_{\text {opt }} \geq \max \left\{S_{1}+\alpha+1+q_{2}, S_{1}+\alpha+2\right\}$. Thus, $L_{\text {on }} / L_{\text {opt }} \leq\left(S_{1}+\alpha+2+q_{2}\right) / \max \left\{S_{1}+\alpha+1+q_{2}, S_{1}+\alpha+\right.$ $2\} \leq 1+1 /(2 \alpha+2)=1+\alpha$, a contradiction. If, in the offline optimal schedule $\pi, J_{2}$ is scheduled in the first starting batch and $r_{2}<S_{1}+\alpha$, then $B_{1}$ is full and the smallest delivery time of jobs in $B_{1}$ is not less than $q_{2}$. Thus $L_{\text {opt }} \geq S_{1}-\alpha+2+q_{2}$, and so $L_{\text {on }} / L_{\text {opt }} \leq\left(S_{1}+\alpha+2+q_{2}\right) /\left(S_{1}-\alpha+2+q_{2}\right) \leq 1+\alpha$, a contradiction.

The above analysis means that $B_{n}$ does not interrupt $B_{n-1}$. Note that $n \geq 2$. If $h=1$, then $S_{n}>S_{n-1}+1$. From S(2) in algorithm $H(b=2)$, we know that $S_{n}=r_{n}$. Thus $L_{\text {on }}=$ $S_{n}+1+q_{n}=r_{n}+1+q_{n} \leq L_{\mathrm{opt}}$, a contradiction. Thus, $h \geq 2$. Claim 1 follows.

Claim 2 (some batch in the $h-1$ batches $B_{n^{\prime}}, B_{n^{\prime}+1}, \ldots, B_{n-1}$ is nonfull). Otherwise, all of the $h-1$ batches $B_{n^{\prime}}, B_{n^{\prime}+1}, \ldots, B_{n-1}$ are full.

If the delivery time of some job in $B_{n^{\prime}}, B_{n^{\prime}+1}, \ldots, B_{n-1}$ is less than $q_{n}$, let $B_{k}$ be the latest starting batch in $B_{n^{\prime}}, B_{n^{\prime}+1}, \ldots, B_{n-1}$ such that some job in $B_{k}$ has a delivery time less than $q_{n}$. Then each job in batches starting in the time interval $\left(S_{k}, S_{n}\right)$ and $J_{n}$ have delivery times not less than $q_{n}$ and then arrive after $S_{k}$. Then $L_{\text {opt }} \geq S_{k}+n-k+q_{n}$, $L_{\text {on }}=S_{k}+n-k+1+q_{n}$, and $L_{\text {on }}-L_{\text {opt }} \leq 1$.

If $k \geq 3$ or $[k=2$ and $n \geq 4]$, then $S_{k}+n-k \geq 2+2 \alpha$, and so $\left(L_{\text {on }}-L_{\text {opt }}\right) / L_{\text {opt }} \leq 1 /(2 \alpha+2)=\alpha$, a contradiction.

If $k=2$ and $n=3$, then we have $r_{3}>S_{2}$ and $L_{\text {opt }} \geq$ $r_{3}+1+q_{3}>S_{2}+1+q_{3}$. As $B_{2}$ is full, there exist at least two batches in the offline optimal schedule $\pi$. Suppose that $B_{2}$ interrupts $B_{1}$, which means that $S_{2}=S_{1}+\alpha$ and $L_{\text {on }}=$ $S_{1}+\alpha+2+q_{3}$. If $J_{3}$ is scheduled in the first starting batch in the offline optimal schedule $\pi$, then $L_{\text {opt }} \geq r_{3}+2 \geq S_{1}+$ $\alpha+2 \geq 2+2 \alpha$. Thus, $\left(L_{\text {on }}-L_{\text {opt }}\right) / L_{\text {opt }} \leq 1 /(2 \alpha+2)=\alpha$, a contradiction. If $J_{3}$ is scheduled after the first starting batch in the offline optimal schedule $\pi$, then $L_{\text {opt }} \geq S_{1}-\alpha+2+q_{3}$. Thus, $L_{\text {on }} / L_{\text {opt }} \leq\left(S_{1}+\alpha+2+q_{3}\right) /\left(S_{1}-\alpha+2+q_{3}\right) \leq 1+\alpha$, a contradiction. So we suppose that $B_{2}$ does not interrupt $B_{1}$; that is, $S_{2} \geq S_{1}+1$. Note that now $L_{\text {on }}=S_{2}+2+q_{3}$. If $S_{2}>S_{1}+1$, then each job in $\left\{B_{2}, B_{3}\right\}$ arrives at or later than $S_{2}$. Considering that $B_{2}$ is full, then $L_{\mathrm{opt}} \geq S_{2}+2 \geq 3+\alpha$. Thus, $\left(L_{\text {on }}-L_{\text {opt }}\right) / L_{\text {opt }} \leq 1 /(\alpha+3)<\alpha$, a contradiction. If $S_{2}=S_{1}+1$, then $L_{\text {on }}=S_{2}+2+q_{3}=S_{1}+3+q_{3}$. If $B_{1}$ is full, then $L_{\text {opt }} \geq 3$. Thus, $\left(L_{\text {on }}-L_{\text {opt }}\right) / L_{\text {opt }} \leq 1 / 3<\alpha$, a contradiction. If $B_{1}$ is nonfull, considering that $B_{2}$ does not interrupt $B_{1}$, then each job in $\left\{B_{2}, B_{3}\right\}$ arrives later than $S_{1}+\alpha$ which means that $L_{\text {opt }} \geq S_{1}+\alpha+2 \geq 2 \alpha+2$. So $\left(L_{\text {on }}-L_{\text {opt }}\right) / L_{\text {opt }} \leq 1 /(2 \alpha+2)=\alpha$, a contradiction.

If $k=1$, noticing that now $B_{1}$ is full and $B_{2}$ does not interrupt $B_{1}$, then each job in batches starting in the time interval $\left(S_{1}, S_{n}\right)$ and $J_{n}$ have delivery times not less than $q_{n}$ and arrive after $S_{1}+\alpha$ by S(3) in algorithm $H(b=2)$. So $L_{\text {opt }} \geq S_{1}+\alpha+n-1+q_{n}$ and $L_{\text {on }}=S_{1}+n+q_{n}$. Thus, $L_{\text {on }} / L_{\text {opt }} \leq$
$\left(S_{1}+n+q_{n}\right) /\left(S_{1}+\alpha+n-1+q_{n}\right) \leq(2+\alpha) /(2 \alpha+1)=1+\alpha$, a contradiction.

So in the following we assume the delivery time of each job in $\left\{B_{n^{\prime}}, B_{n^{\prime}+1}, \ldots, B_{n-1}\right\}$ is not less than $q_{n}$. Then $L_{\text {opt }} \geq$ $S_{1}-\alpha+h+q_{n}$.

If $B_{n^{\prime}}$ interrupts $B_{n^{\prime}-1}$, then $n^{\prime}=2,3$. If $n^{\prime}=2$, then $L_{\text {on }}=$ $S_{2}+h+q_{n}=S_{1}+\alpha+h+q_{n}$. Considering $h \geq 2$ and $S_{1} \geq \alpha$, we have $L_{\text {on }} / L_{\text {opt }} \leq\left(S_{1}+\alpha+h+q_{n}\right) /\left(S_{1}-\alpha+h+q_{n}\right) \leq 1+\alpha$, a contradiction. If $n^{\prime}=3$, then $B_{2}$ is nonfull, $S_{2} \geq S_{1}+1$, and $L_{\mathrm{on}}=S_{3}+h+q_{n}=S_{2}+\alpha+h+q_{n}$. Note that now there is only one job in $B_{2}$. If $q_{2}<q_{n}$, considering jobs in the set of $\left\{B_{n^{\prime}}, B_{n^{\prime}+1}, \ldots, B_{n-1}, J_{n}\right\}$ all have delivery times not less than $q_{n}$, then these jobs arrive after $S_{2}$, and, accordingly, $L_{\text {opt }} \geq$ $S_{2}+h+q_{n}$. So $L_{\text {on }} / L_{\text {opt }} \leq\left(S_{2}+\alpha+h+q_{n}\right) /\left(S_{2}+h+q_{n}\right)<1+\alpha$, a contradiction. If $q_{2} \geq q_{n}$ and $r_{2}<S_{1}+\alpha$, then $S_{2}=S_{1}+1$, $L_{\text {on }}=S_{1}+\alpha+h+1+q_{n}, B_{1}$ is full, and the delivery time of any job in $B_{1}$ is not less than $q_{n}$. And so $L_{\text {opt }} \geq S_{1}-\alpha+h+1+q_{n}$. So $L_{\text {on }} / L_{\text {opt }} \leq\left(S_{1}+\alpha+h+1+q_{n}\right) /\left(S_{1}-\alpha+h+1+q_{n}\right)<1+\alpha$, a contradiction. If $q_{2} \geq q_{n}$ and $S_{1}+\alpha \leq r_{2} \leq S_{1}+1$, considering that there is only one job in $B_{2}$, then $S_{2}=S_{1}+1$ and $L_{\text {on }}=$ $S_{1}+\alpha+h+1+q_{n}$, and each job in $\left\{B_{n^{\prime}}, B_{n^{\prime}+1}, \ldots, B_{n}\right\}$ arrives at or after $S_{1}+\alpha$. So $L_{\text {opt }} \geq S_{1}+\alpha+h+q_{n}$. Thus $L_{\text {on }} / L_{\text {opt }} \leq$ $\left(S_{1}+\alpha+h+1+q_{n}\right) /\left(S_{1}+\alpha+h+q_{n}\right) \leq 1+1 /(2+2 \alpha)=1+\alpha$, a contradiction. If $q_{2} \geq q_{n}$ and $r_{2}>S_{1}+1$, then $S_{2}>S_{1}+1$ and each job in $\left\{B_{n^{\prime}}, B_{n^{\prime}+1}, \ldots, B_{n}\right\}$ arrives at or after $S_{2}$, and so $L_{\text {opt }} \geq S_{2}+h+q_{n}$. Thus, $L_{\text {on }} / L_{\text {opt }} \leq\left(S_{2}+\alpha+h+q_{n}\right) /\left(S_{2}+\right.$ $\left.h+q_{n}\right) \leq 1+\alpha$, a contradiction.

If $n^{\prime}=1$ or $\left[n^{\prime} \geq 2\right.$ and $B_{n^{\prime}}$ does not interrupt $\left.B_{n^{\prime}-1}\right]$, then there exists some time instant $t^{\prime}$ such that $t^{\prime}<S_{n^{\prime}}$ and the time interval $\left[t^{\prime}, S_{n^{\prime}}\right)$ is an idle time in $\sigma$. Then by $S(1)$, $S(2)$ in algorithm $H(b=2)$, the arrival time of any job in $\left\{B_{n^{\prime}}, B_{n^{\prime}+1}, \ldots, B_{n}\right\}$ is not less than $S_{n^{\prime}}-\alpha$. Thus $L_{\text {opt }} \geq S_{n^{\prime}}-\alpha+$ $h+q_{n}$, and so $L_{\text {on }} / L_{\text {opt }} \leq\left(S_{n^{\prime}}+h+q_{n}\right) /\left(S_{n^{\prime}}-\alpha+h+q_{n}\right)<1+\alpha$, a contradiction. Claim 2 follows.

Let $e \leq n-1$ be maximum so that $B_{e}$ is a nonfull batch. From Claim 2, we have $e \geq n^{\prime}$.

Claim $3(e \leq n-2)$. Otherwise, suppose that $e=n-1$. Since $B_{n-1}$ is nonfull, we have $r_{n}>S_{n-1}, L_{\text {on }}=S_{e}+2+q_{n}$, and $L_{\text {opt }} \geq r_{n}+1+q_{n}>S_{e}+1+q_{n}$. If some batch starting before $S_{e}$ is interrupted, noticing that $B_{e}$ is a nonfull batch, then the interrupted batch must start before $S_{e-1}$, and then $S_{e} \geq S_{3} \geq$ $2 \alpha+1$. Hence, $L_{\text {on }} / L_{\text {opt }} \leq\left(S_{e}+2+q_{n}\right) /\left(S_{e}+1+q_{n}\right) \leq 1+1 /(2 \alpha+$ $2)=1+\alpha$, a contradiction. So in the following we assume that each batch starting before $S_{e}$ is not interrupted. If $n \leq 3$, then $e \leq 2$. As $B_{e}$ is a nonfull batch and not interrupted, by $\mathrm{S}(3.1)$ in algorithm $H(b=2)$, all jobs in $B_{n}$ arrive after $S_{e}+\alpha$, which means that $L_{\text {opt }} \geq S_{e}+\alpha+1+q_{n}$. Hence, $L_{\text {on }} / L_{\text {opt }} \leq$ $\left(S_{e}+2+q_{n}\right) /\left(S_{e}+\alpha+1+q_{n}\right) \leq(2+\alpha) /(2 \alpha+1)=1+\alpha$, a contradiction. If $n \geq 4$, then $S_{e} \geq S_{3} \geq 1+2 \alpha$, and accordingly, $L_{\text {on }} / L_{\text {opt }} \leq\left(S_{e}+2+q_{n}\right) /\left(S_{e}+1+q_{n}\right) \leq 1+1 /(2 \alpha+2)=1+\alpha$, a contradiction. Claim 3 follows.

By the definition of $B_{e}$ and Claim $3, n-e \geq 2$, $B_{e+1}, \ldots, B_{n-1}$ are all full and each job in batches starting after $S_{e}$ arrives after $S_{e}$. And $L_{\text {on }}=S_{e}+n-e+1+q_{n}$.

If each job in $B_{e+1}, \ldots, B_{n-1}$ has a delivery time not less than $q_{n}$, then $L_{\text {opt }} \geq S_{e}+n-e+q_{n}$. If $e \geq 2$, then $S_{e} \geq 2 \alpha$. Then $L_{\text {on }} / L_{\text {opt }} \leq\left(S_{e}+n-e+1+q_{n}\right) /\left(S_{e}+n-e+q_{n}\right) \leq$ 
$1+1 /(2 \alpha+2)=1+\alpha$, a contradiction. If $e=1$, considering $B_{e}$ is nonfull and not interrupted, then, by S(3.1) in algorithm $H(b=2)$, each job in batches starting after $S_{1}$ arrives after $S_{1}+\alpha$. Thus $L_{\text {opt }} \geq S_{1}+\alpha+n-1+q_{n}$. Hence, $L_{\text {on }} / L_{\text {opt }} \leq$ $\left(S_{1}+n+q_{n}\right) /\left(S_{1}+\alpha+n-1+q_{n}\right) \leq(3+\alpha) /(2 \alpha+2)<1+\alpha$, a contradiction.

So in the following we assume that some job in $B_{e+1}, \ldots, B_{n-1}$ has a delivery time less than $q_{n}$. Let $e+1 \leq$ $k^{\prime} \leq n-1$ be maximum so that some job in $B_{k^{\prime}}$ has a delivery time less than $q_{n}$. Then $k^{\prime} \geq 2$ and batches starting in the time interval $\left(S_{k^{\prime}}, S_{n}\right)$ are all full. Also each job in batches starting in the time interval $\left(S_{k^{\prime}}, S_{n}\right)$ and $J_{n}$ have delivery times not less than $q_{n}$ and arrive after $S_{k^{\prime}}$. Thus $L_{\text {opt }} \geq S_{k^{\prime}}+n-k^{\prime}+q_{n}$. Noting that $L_{\text {on }}=S_{k^{\prime}}+n-k^{\prime}+1+q_{n}$, we have $L_{\text {on }}-L_{\text {opt }} \leq 1$. If $k^{\prime} \geq 3$, then $S_{k^{\prime}} \geq 1+2 \alpha$. Hence, $L_{\text {on }} / L_{\text {opt }} \leq\left(S_{k^{\prime}}+n-k^{\prime}+1+\right.$ $\left.q_{n}\right) /\left(S_{k^{\prime}}+n-k^{\prime}+q_{n}\right) \leq 1+1 /(2 \alpha+2)=1+\alpha$, a contradiction. If $k^{\prime}=2$, then $e=1, n \geq 3$, and $S_{k^{\prime}} \geq 2 \alpha$. As now $B_{1}$ is nonfull and not interrupted, then by $\mathrm{S}(3.1)$ in algorithm $H(b=2)$, each job in batches starting after $S_{1}$ arrives after $S_{1}+\alpha$. Thus $L_{\text {opt }} \geq S_{1}+\alpha+2 \geq 2 \alpha+2$. So $\left(L_{\text {on }}-L_{\text {opt }}\right) / L_{\text {opt }} \leq 1 /(2 \alpha+2)=\alpha$, a contradiction.

From the above discussion we conclude that algorithm $H(b=2)$ has a competitive ratio of at most $1+\alpha$. By Theorem 1, we know that algorithm $H(b=2)$ is an optimal online algorithm with a competitive ratio of $1+\alpha$. This completes the proof.

\section{Problem P-Batch $(b \geq 3)$}

Note that $\beta$ is the positive solution of $\beta(1+\beta)^{2}=1$.

Theorem 3. For problem P-batch $(b \geq 3)$, there exists no online algorithm with a competitive ratio of less than $1+\beta$.

Proof. Theorem 2.4 in Liu and Yuan [21] shows that, for problem 1 online, $r_{j}, p_{j}=1$, P-batch, $b<\infty$, L-restart | $C_{\max }$ with $b \geq 3$, no online algorithm has a competitive ratio of less than $1+\beta$. Here $C_{\max }$ is makespan, namely, the maximum completion time of all jobs on the parallel batch machine. Problem $1 \mid$ online, $r_{j}, p_{j}=1$, P-batch, $b<$ $\infty$, L-restart $\mid C_{\max }$ is the special case of $1 \mid$ online, $r_{j}, p_{j}=$ 1, P-batch, $b<\infty$, L-restart $\mid L_{\max }$. So for problem P-batch $(b \geq 3)$, no online algorithm has a competitive ratio of less than $1+\beta$. The theorem follows.

Algorithm $H(b \geq 3)$. Initially set $k=0$. At the current time instant $t$, do the following.

$\mathrm{S}(1)$ : if $k=0, U(t) \neq \emptyset$, and $t \geq \beta$, then select $\min \{b,|U(t)|\}$ jobs in $U(t)$ such that each job selected has a delivery time not less than the largest delivery time of jobs not selected in $U(t)$ as the batch $B_{k+1}$ starting at time $t$. Then reset $k$ to be $k+1$.

$\mathrm{S}(2)$ : if $k \geq 1$, the machine is idle, and $U(t) \neq \emptyset$, select $\min \{b,|U(t)|\}$ jobs in $U(t)$ such that each job selected has a delivery time not less than the largest delivery time of jobs not selected in $U(t)$ as the batch $B_{k+1}$ starting at time $t$. Then reset $k$ to be $k+1$.
$S(3)$ : if $S_{1}<1 / \beta-1, U(t) \neq \emptyset$, the machine is occupied by $B_{k}, k \in\{1,2\}$, and each batch starting earlier than $S_{k}$ is not interrupted, do the following.

$\mathrm{S}(3.1)$ : if $k=1, B_{k}$ is nonfull, $t=(1+\beta) S_{k}+\beta$, and $\left|B_{k}\right|+|U(t)| \leq b$, then let $q^{\prime}$ be the largest delivery time of jobs in $U(t)$. If $q^{\prime} \geq q_{1}$, or $q^{\prime} \geq \beta$, or $\left[q^{\prime}<\beta\right.$, and $\left.q_{1}<1-\beta\right]$, then do Restart $(k, t)$.

$\mathrm{S}(3.2)$ : if $k=1, B_{k}$ is full, $t=S_{k}+\beta$, the largest delivery time of jobs in $U(t)$ is not less than $\beta^{2}+\beta$, and the delivery time of some job in $B_{1}$ is less than the delivery time of some job in $U(t)$, then do Restart $(k, t)$.

$\mathrm{S}(3.3)$ : if $k=2, B_{k}$ is nonfull, $t=S_{k}+\beta,\left|B_{k}\right|+|U(t)| \leq b$, and \{either $B_{1}$ is full or $\left[B_{1}\right.$ is nonfull and the number of jobs arriving in the time interval $\left[0,(1+\beta) S_{1}+\beta\right]$ is not more than $b]\}$, then do Restart $(k, t)$.

$S(4)$ : otherwise, do nothing but wait.

From algorithm $H(b \geq 3)$ we have the following observation.

Observation 2. (1) The earliest arrival time of all jobs is not less than $S_{1}-\beta$. If $S_{1}>\beta$, then all jobs arrive at or after $S_{1}$.

(2) If $S_{1} \geq 1 / \beta-1$ or $\left\{S_{1}<1 / \beta-1, B_{1}\right.$ is nonfull, and the number of jobs arriving in the time interval $\left[0,(1+\beta) S_{1}+\beta\right]$ is more than $b\}$, then there exists no batch which is interrupted.

(3) If $k \geq 3$, then $S_{k} \geq 2 \beta+1$.

(4) If $S_{1}<1 / \beta-1$, then $(1+\beta) S_{1}+\beta<S_{1}+1$.

Theorem 4. For problem P-batch $(b \geq 3), H(b \geq 3)$ is a best possible online algorithm with a competitive ratio of $1+\beta$.

Proof. Suppose to the contrary that $I$ is an instance so that $L_{\text {on }} / L_{\text {opt }}>1+\beta$. Let $\sigma$ be the schedule generated by running algorithm $H(b \geq 3)$ for the instance $I$ and let $\pi$ be an offline optimal schedule for the instance $I$. As the proof in Theorem 2, $q_{i}$ is used to denote the largest delivery time of jobs in $B_{i}$ and $r_{i}$ is the latest arrival time of jobs whose delivery times are $q_{i}$ in $B_{i}$. Let $B_{n}$ be the earliest starting batch in $\sigma$ which is not interrupted and assumes $L_{\text {on }}$. Then $L_{\text {on }}=$ $S_{n}+1+q_{n}$. As $S_{1} \leq \max \left\{r_{1}, \beta\right\}$, then the assumption that $L_{\text {on }} / L_{\text {opt }}>1+\beta$ implies $n \geq 2$.

Let $C_{\text {on }}=S_{n}+1$; that is, $C_{\text {on }}$ is the completion time of $B_{n}$ on the parallel batch machine. Let $t$ be the minimum time instant such that, in $\sigma$, there are no idle times between $t$ and $C_{\text {on }}$ and each batch processed in the time interval $\left[t, C_{\text {on }}\right]$ is not interrupted. Suppose that there are $h$ batches $B_{n-h+1}, \ldots, B_{n}$ processed in the time interval $\left[t, C_{\text {on }}\right]$ in $\sigma$. Then $C_{\text {on }}=t+h$. Set $n^{\prime}=n-h+1$. So $S_{n^{\prime}}=t$.

Claim 1 ( $B_{n}$ does not interrupt $B_{n-1}$ and $\left.h \geq 2\right)$. Otherwise, $B_{n}$ is a restricted batch. By algorithm $H(b \geq 3)$, we have $n=$ 2,3 and $S_{1}<1 / \beta-1$; that is, $(1+\beta) S_{1}+\beta<S_{1}+1$. Let $q^{\prime \prime}$ be the largest delivery time of jobs arriving in the interval $\left(S_{n-1}, S_{n}\right]$, let $J^{\prime \prime}$ be the job with delivery time $q^{\prime \prime}$, and let $r^{\prime \prime}$ be the arrival time of $J^{\prime \prime}$. Then $q_{n}=\max \left\{q_{n-1}, q^{\prime \prime}\right\}$. If $q^{\prime \prime} \geq q_{n-1}$, then $q_{n}=q^{\prime \prime}, L_{\text {opt }} \geq S_{n-1}+1+q^{\prime \prime}, L_{\text {on }}=S_{n}+1+q^{\prime \prime} \leq$ $(1+\beta) S_{n-1}+\beta+1+q^{\prime \prime}$, and, accordingly, $L_{\text {on }} / L_{\text {opt }} \leq 1+\beta$, a contradiction. So in the following we assume that $q^{\prime \prime}<q_{n-1}$; that is, $q_{n}=q_{n-1}$. Then $L_{\text {on }}=S_{n}+1+q_{n-1}$. If, in the offline optimal schedule $\pi$, there exists only one batch, or $J_{n-1}$ and $J^{\prime \prime}$ 
are scheduled in one batch, or the batch containing $J_{n-1}$ starts later than the batch containing $J^{\prime \prime}$, then $L_{\text {opt }} \geq S_{n-1}+1+q_{n-1}$. Considering $L_{\text {on }}=S_{n}+1+q_{n-1} \leq(1+\beta) S_{n-1}+\beta+1+q_{n-1}$, then $L_{\text {on }} / L_{\text {opt }} \leq 1+\beta$, a contradiction. So in the following we assume that, in the offline optimal schedule $\pi$, there exist at least 2 batches, $J_{n-1}$ and $J^{\prime \prime}$ are scheduled in two different batches, and the batch containing $J_{n-1}$ starts earlier than the batch containing $J^{\prime \prime}$. Then we easily get $L_{\text {opt }} \geq \max \left\{r_{n-1}+1+\right.$ $\left.q_{n-1}, r_{n-1}+2+q^{\prime \prime}\right\} \geq S_{1}-\beta+2+q^{\prime \prime}$.

If $n=2$ and $B_{1}$ is full, then $q^{\prime \prime}<q_{1}, S_{2}=S_{1}+\beta$, and $L_{\text {on }}=$ $S_{1}+\beta+1+q_{1}$. By Observation 2 , we have $L_{\text {opt }} \geq S_{1}-\beta+1+q_{1}$. Considering $L_{\text {opt }} \geq 2$, then $\left(L_{\text {on }}-L_{\text {opt }}\right) / L_{\text {opt }} \leq(2 \beta) / 2=\beta$, a contradiction.

If $n=2$ and $B_{1}$ is nonfull, then $L_{\text {on }}=(1+\beta) S_{1}+\beta+1+q_{1}$. If $q_{1}<1-\beta$, then $L_{\text {on }}<(1+\beta) S_{1}+2$. Noticing that $L_{\text {opt }} \geq$ $S_{1}-\beta+2$, then $L_{\text {on }} / L_{\text {opt }} \leq\left((1+\beta) S_{1}+2\right) /\left(S_{1}-\beta+2\right)<1+\beta$, a contradiction. Hence we have $q_{1} \geq 1-\beta$. As $B_{2}$ interrupts $B_{1}$, considering $q^{\prime \prime}<q_{1}$ and $q_{1} \geq 1-\beta$, then, by $S(3.1)$ in algorithm $H(b \geq 3)$, the only possibility is that $q^{\prime \prime} \geq \beta$. Then $L_{\mathrm{opt}} \geq S_{1}-\beta+2+q^{\prime \prime} \geq S_{1}+2$. Thus, $L_{\mathrm{on}} / L_{\mathrm{opt}} \leq\left((1+\beta) S_{1}+\beta+\right.$ $\left.1+q_{1}\right) / \max \left\{S_{1}-\beta+1+q_{1}, S_{1}+2\right\} \leq 1+\left(\beta\left(S_{1}+2\right)\right) /\left(S_{1}+2\right)=$ $1+\beta$, a contradiction.

If $n=3$, then $S_{3}=S_{2}+\beta$ and $L_{\text {on }}=S_{2}+\beta+1+q_{2}$. Also noticing that now $B_{2}$ does not interrupt $B_{1}$, we have $S_{2} \geq$ $S_{1}+1$. Note that $q^{\prime \prime}<q_{n-1}$ and in the offline optimal schedule $\pi$, the batch containing $J_{n-1}=J_{2}$ starts earlier than the batch containing $J^{\prime \prime}$. If $r_{2} \leq S_{1}$, then $S_{2}=S_{1}+1, L_{\text {on }}=S_{1}+\beta+2+q_{2}$, $B_{1}$ is full and each job in $B_{1}$ has a delivery time not less that $q_{2}$, and, accordingly, $L_{\mathrm{opt}} \geq S_{1}-\beta+2+q_{2}$. Thus, $L_{\mathrm{on}} / L_{\mathrm{opt}} \leq\left(S_{1}+\right.$ $\left.\beta+2+q_{2}\right) /\left(S_{1}-\beta+2+q_{2}\right) \leq 1+\beta$, a contradiction. If $r_{2} \geq S_{2}$, then $L_{\text {opt }} \geq S_{2}+1+q_{2}$. Thus $L_{\text {on }} / L_{\text {opt }} \leq\left(S_{2}+\beta+1+q_{2}\right) /\left(S_{2}+\right.$ $\left.1+q_{2}\right)<1+\beta$, a contradiction. If $(1+\beta) S_{1}+\beta \leq r_{2}<S_{2}$, then $S_{2}=S_{1}+1, L_{\text {on }}=S_{1}+\beta+2+q_{2}$, and $L_{\text {opt }} \geq(1+\beta) S_{1}+\beta+1+q_{2}$. Thus $L_{\text {on }} / L_{\text {opt }}<\left(S_{1}+\beta+2+q_{2}\right) /\left((1+\beta) S_{1}+\beta+1+q_{2}\right) \leq$ $\left(2 \beta+2+q_{2}\right) /\left((1+\beta) \beta+\beta+1+q_{2}\right)<1+\beta$, a contradiction. So in the following we assume that $S_{1}<r_{2}<(1+\beta) S_{1}+\beta$. Then $S_{2}=S_{1}+1, L_{\text {on }}=S_{1}+\beta+2+q_{2}$, and $L_{\text {opt }} \geq r_{2}+2>S_{1}+2$. If $q_{2} \leq \beta+\beta^{2}$, then $L_{\text {on }} / L_{\text {opt }}<\left(S_{1}+\beta+2+\beta+\beta^{2}\right) /\left(S_{1}+2\right) \leq$ $1+\beta$, a contradiction. If $q_{2}>\beta+\beta^{2}$, noticing that $B_{2}$ does not interrupt $B_{1}$ and $B_{3}$ interrupts $B_{2}$, by $\mathrm{S}(3)$ in algorithm $H(b \geq 3)$, then $B_{1}$ is full. If each job in the full batch $B_{1}$ has a delivery time not less than $q_{2}$, then $L_{\mathrm{opt}} \geq S_{1}-\beta+2+q_{2}$, and then $L_{\text {on }} / L_{\text {opt }} \leq\left(S_{1}+\beta+2+q_{2}\right) /\left(S_{1}-\beta+2+q_{2}\right) \leq 1+\beta$, a contradiction. So some job in the full batch $B_{1}$ has a delivery time less than $q_{2}$. As $B_{1}$ is full, $q_{2}>\beta+\beta^{2}$, some job in $B_{1}$ has a delivery time less than $q_{2}$, and $B_{2}$ does not interrupt $B_{1}$, then $r_{2}>S_{1}+\beta$, and so $L_{\text {opt }} \geq \max \left\{S_{1}+\beta+1+q_{2}, S_{1}+\beta+2\right\}$. Thus, $L_{\text {on }} / L_{\text {opt }}<\left(S_{1}+\beta+2+q_{2}\right) / \max \left\{S_{1}+\beta+1+q_{2}, S_{1}+\beta+2\right\} \leq$ $1+1 /(2 \beta+2)<1+\beta$, a contradiction.

The above analysis means that $B_{n}$ does not interrupt $B_{n-1}$. Note that $n \geq 2$. If $h=1$, then $S_{n}>S_{n-1}+1$. From S(2) in algorithm $H(b \geq 3)$, we know that $S_{n}=r_{n}$. Thus $L_{\text {on }}=$ $S_{n}+1+q_{n}=r_{n}+1+q_{n} \leq L_{\text {opt }}$, a contradiction. Hence, $h \geq 2$. Claim 1 follows.

Claim 2 (some batch in the $h-1$ batches $B_{n^{\prime}}, B_{n^{\prime}+1}, \ldots, B_{n-1}$ is nonfull). Otherwise, all of the $h-1$ batches $B_{n^{\prime}}, B_{n^{\prime}+1}, \ldots, B_{n-1}$ are full.
If the smallest delivery time of jobs in $B_{n^{\prime}}, B_{n^{\prime}+1}, \ldots, B_{n-1}$ is not less than $q_{n}$ and $B_{n^{\prime}}$ interrupts $B_{n^{\prime}-1}$, then $n^{\prime}=2,3$. If $n^{\prime}=2$ and $B_{1}$ is full, then $L_{\text {on }}=S_{n^{\prime}}+h+q_{n}=S_{1}+\beta+h+q_{n}$ and $L_{\text {opt }} \geq S_{1}-\beta+h+q_{n}$. Thus, considering $h \geq 2$ and $S_{1} \geq \beta$, we have $L_{\text {on }} / L_{\text {opt }} \leq\left(S_{1}+\beta+h+q_{n}\right) /\left(S_{1}-\beta+h+q_{n}\right) \leq$ $1+\beta$, a contradiction. If [ $n^{\prime}=2$ and $B_{1}$ is nonfull $]$ or $n^{\prime}=3$, then $B_{n^{\prime}-1}$ is nonfull and each job in batches starting after $S_{n^{\prime}}$ arrives after $S_{n^{\prime}}$. Then $L_{\text {opt }} \geq S_{n^{\prime}}+h-1+q_{n}$ and $S_{n^{\prime}} \geq \min \{(1+$ $\left.\beta) S_{1}+\beta, S_{1}+1+\beta\right\} \geq \min \left\{(1+\beta)^{2}-1,1+2 \beta\right\} \geq(1+\beta)^{2}-1$. Thus $L_{\text {on }} / L_{\text {opt }} \leq\left(S_{n^{\prime}}+h+q_{n}\right) /\left(S_{n^{\prime}}+h-1+q_{n}\right) \leq 1+1 /(1+\beta)^{2}=1+\beta$, a contradiction.

If the smallest delivery time of jobs in $B_{n^{\prime}}, B_{n^{\prime}+1}, \ldots, B_{n-1}$ is not less than $q_{n}$ and $\left\{n^{\prime}=1\right.$ or $\left[n^{\prime} \geq 2\right.$ and $B_{n^{\prime}}$ does not interrupt $\left.\left.B_{n^{\prime}-1}\right]\right\}$, then the arrival time of any job in $B_{n^{\prime}}, B_{n^{\prime}+1}, \ldots, B_{n}$ is not less than $S_{n^{\prime}}-\beta$. Thus $L_{\text {opt }} \geq S_{n^{\prime}}-\beta+$ $h+q_{n}$, and so $L_{\text {on }} / L_{\text {opt }} \leq\left(S_{n^{\prime}}+h+q_{n}\right) /\left(S_{n^{\prime}}-\beta+h+q_{n}\right)<1+\beta$, a contradiction.

So in the following we suppose that the delivery time of some job in $B_{n^{\prime}}, B_{n^{\prime}+1}, \ldots, B_{n-1}$ is less than $q_{n}$. Let $B_{k}$ be the latest starting batch in $B_{n^{\prime}}, B_{n^{\prime}+1}, \ldots, B_{n-1}$ such that some job in $B_{k}$ has a delivery time less than $q_{n}$. Then each job in batches starting in the time interval $\left(S_{k}, S_{n}\right)$ and $J_{n}$ have delivery times not less than $q_{n}$ and, accordingly, arrive after $S_{k}$. Then $L_{\text {opt }} \geq$ $S_{k}+n-k+q_{n}, L_{\text {on }}=S_{k}+n-k+1+q_{n}$, and $L_{\text {on }}-L_{\text {opt }} \leq 1$.

If $k \geq 3$ or $[k=2$ and $n \geq 4]$, then $L_{\text {opt }} \geq S_{k}+n-k \geq$ $2+2 \beta$, and, accordingly, $\left(L_{\text {on }}-L_{\text {opt }}\right) / L_{\text {opt }} \leq 1 /(2+2 \beta)<\beta$, a contradiction.

If $k=2, n=3$, and $\left\{B_{1}\right.$ is nonfull or $\left[B_{1}\right.$ is full and $B_{2}$ does not interrupt $\left.\left.B_{1}\right]\right\}$, then $S_{2} \geq \min \left\{(1+\beta) S_{1}+\beta, S_{1}+1\right\} \geq$ $(1+\beta)^{2}-1$. Thus, $\left(L_{\text {on }}-L_{\text {opt }}\right) / L_{\text {opt }} \leq 1 /(1+\beta)^{2}=\beta$, a contradiction. If $k=2, n=3, B_{1}$ is full, and $B_{2}$ interrupts $B_{1}$, then some job with a delivery time not less than $\beta^{2}+\beta$ arrives after $S_{1}$ and $L_{\text {opt }} \geq S_{1}+1+\beta^{2}+\beta \geq(1+\beta)^{2}$. Thus, $\left(L_{\text {on }}-L_{\text {opt }}\right) / L_{\text {opt }} \leq 1 /(1+\beta)^{2}=\beta$, a contradiction.

If $k=1$ and $n \geq 3$, then $L_{\text {opt }} \geq S_{k}+n-k \geq 2+\beta$, and, accordingly, $\left(L_{\text {on }}-L_{\text {opt }}\right) / L_{\text {opt }} \leq 1 /(\beta+2)<\beta$, a contradiction. If $k=1$ and $n=2$, then there are at least two batches in the offline optimal schedule $\pi$. Note that now $r_{2}>S_{1}$. If $J_{n}=J_{2}$ is scheduled in the first batch in the offline optimal schedule $\pi$, then $L_{\text {opt }} \geq \max \left\{S_{1}+1+q_{2}, S_{1}+2\right\} \geq 2+\beta$. Thus, $\left(L_{\text {on }}-L_{\text {opt }}\right) / L_{\text {opt }} \leq 1 /(\beta+2)<\beta$, a contradiction. If $J_{n}=J_{2}$ is scheduled in the batch starting after the first batch in the offline optimal schedule $\pi$, then $L_{\text {opt }} \geq S_{1}-\beta+2+q_{2}$. Thus, $L_{\text {on }} / L_{\text {opt }} \leq\left(S_{1}+2+q_{2}\right) /\left(S_{1}-\beta+2+q_{2}\right) \leq 1+\beta$, a contradiction. Claim 2 follows.

Let $e \leq n-1$ be maximum so that $B_{e}$ is a nonfull batch. From Claim 2, we have $k \geq n^{\prime}$.

Claim 3 (e $\leq n-2)$. Otherwise, suppose that $e=n-1$. As $B_{e}$ is a nonfull batch, then $r_{n}>S_{e}, L_{\text {on }}=S_{e}+2+q_{n}$, and $L_{\text {opt }} \geq r_{n}+1+q_{n}>S_{e}+1+q_{n}$.

If $e \geq 2$, noticing that $B_{e}$ is a nonfull batch, then $S_{e} \geq$ $\min \left\{(1+\beta) S_{1}+\beta, S_{1}+1\right\} \geq(1+\beta)^{2}-1$. Then $L_{\text {on }} / L_{\text {opt }} \leq$ $\left(S_{e}+2+q_{n}\right) /\left(S_{e}+1+q_{n}\right) \leq 1+1 /(1+\beta)^{2}=1+\beta$, a contradiction.

If $e=1$, then $n=2, r_{2}>S_{1}, L_{\text {on }}=S_{1}+2+q_{2}$, and $L_{\text {opt }}>S_{1}+1+q_{2}$. If $S_{1} \geq 1 / \beta-1$, then $L_{\text {on }} / L_{\text {opt }} \leq\left(S_{1}+\right.$ $\left.2+q_{2}\right) /\left(S_{1}+1+q_{2}\right) \leq 1+1 /(1 / \beta)=1+\beta$, a contradiction. 
So we suppose that $S_{1}<1 / \beta-1$. If $r_{2} \geq(1+\beta) S_{1}+\beta$, then $L_{\text {opt }} \geq r_{2}+1+q_{2} \geq(1+\beta)^{2}$. Thus $L_{\text {on }} / L_{\text {opt }} \leq 1+1 /(1+\beta)^{2}=$ $1+\beta$, a contradiction. So in the following we assume that $r_{2}<(1+\beta) S_{1}+\beta$. If there is only one batch in the offline optimal schedule $\pi$, then $\left|B_{1}\right|+\left|B_{2}\right| \leq b$ and $L_{\text {opt }} \geq S_{1}+1+q_{1}$. Also considering that $B_{2}$ does not interrupt $B_{1}$, we have that $q_{1} \geq 1-\beta$ and $q_{2}<\beta$. Then $L_{\mathrm{opt}} \geq S_{1}+1+q_{1} \geq S_{1}+2-\beta$. And $L_{\text {on }} / L_{\text {opt }} \leq\left(S_{1}+2+q_{2}\right) /\left(S_{1}+1+q_{1}\right) \leq\left(S_{1}+2+\beta\right) /\left(S_{1}+2-\beta\right) \leq$ $1+\beta$, a contradiction. So in the following we assume that there are at least two batches in the offline optimal schedule $\pi$. If $J_{n}=J_{2}$ is scheduled in the first starting batch in the offline optimal schedule $\pi$, then $L_{\text {opt }} \geq \max \left\{S_{1}+1+q_{2}, S_{1}+2\right\} \geq$ $2+\beta$. Thus, $L_{\text {on }} / L_{\text {opt }} \leq 1+1 /(\beta+2)<1+\beta$. If $J_{n}=J_{2}$ is scheduled in the batch starting after the first starting batch in the offline optimal schedule $\pi$, then $L_{\text {opt }} \geq S_{1}-\beta+2+q_{2}$. Thus, $L_{\text {on }} / L_{\text {opt }} \leq\left(S_{1}+2+q_{2}\right) /\left(S_{1}-\beta+2+q_{2}\right) \leq 1+\beta$, a contradiction. Claim 3 follows.

By the definition of $B_{e}$ and Claim $3, n-e \geq 2$, $B_{e+1}, \ldots, B_{n-1}$ are all full and each job in batches starting after $S_{e}$ arrives after $S_{e}$. And $L_{\text {on }}=S_{e}+n-e+1+q_{n}$.

If each job in $B_{e+1}, \ldots, B_{n-1}$ has a delivery time not less than $q_{n}$, then $L_{\text {opt }} \geq S_{e}+n-e+q_{n} \geq 2+\beta$. Then $L_{\text {on }} / L_{\text {opt }} \leq$ $\left(S_{e}+n-e+1+q_{n}\right) /\left(S_{e}+n-e+q_{n}\right) \leq 1+1 /(\beta+2)<1+\beta$, a contradiction. So in the following we assume that some job in $B_{e+1}, \ldots, B_{n-1}$ has a delivery time less than $q_{n}$. Let $e+1 \leq$ $k^{\prime} \leq n-1$ be maximum so that some job in $B_{k^{\prime}}$ has a delivery time less than $q_{n}$. Then $k^{\prime} \geq 2$ and batches starting in the time interval $\left(S_{k^{\prime}}, S_{n}\right)$ are all full. And each job in batches starting in the time interval $\left(S_{k^{\prime}}, S_{n}\right)$ and $J_{n}$ have delivery times not less than $q_{n}$ and then arrive after $S_{k^{\prime}}$. Thus $L_{\text {opt }} \geq S_{k^{\prime}}+n-k^{\prime}+q_{n}$. Note that $L_{\text {on }}=S_{k^{\prime}}+n-k^{\prime}+1+q_{n}$. Considering $k^{\prime} \geq 2$, then $S_{k^{\prime}} \geq S_{e}+1 \geq 1+\beta$. Hence, $L_{\text {on }} / L_{\text {opt }} \leq\left(S_{k^{\prime}}+n-k^{\prime}+1+\right.$ $\left.q_{n}\right) /\left(S_{k^{\prime}}+n-k^{\prime}+q_{n}\right) \leq 1+1 /(\beta+2)<1+\beta$, a contradiction.

From the above discussion we conclude that algorithm $H(b \geq 3)$ has a competitive ratio of at most $1+\beta$. By Theorem 3 we know that algorithm $H(b \geq 3)$ is an optimal online algorithm with a competitive ratio of $1+\beta$. This completes the proof.

\section{Conflict of Interests}

The authors declare that there is no conflict of interests regarding the publication of this paper.

\section{Acknowledgments}

This work was supported by the Natural Sciences Foundation (Grant no. 20142BAB211017) of Jiangxi Province and the School Subject (Grant no. 06162015) of Jiangxi University of Finance and Economics.

\section{References}

[1] H. Hoogeveen, C. N. Potts, and G. J. Woeginger, "On-line scheduling on a single machine: maximizing the number of early jobs," Operations Research Letters, vol. 27, no. 5, pp. 193197, 2000.
[2] M. van den Akker, H. Hoogeveen, and N. Vakhania, "Restarts can help in the on-line minimization of the maximum delivery time on a single machine," Journal of Scheduling, vol. 3, no. 6, pp. 333-341, 2000.

[3] L. Epstein and R. van Stee, "Lower bounds for on-line singlemachine scheduling," Theoretical Computer Science, vol. 299, no. 1, pp. 439-450, 2003.

[4] R. van Stee and H. La Poutré, "Minimizing the total completion time on-line on a single machine, using restarts," Journal of Algorithms, vol. 57, no. 2, pp. 95-129, 2005.

[5] J. J. Yuan, R. Y. Fu, C. T. Ng, and T. C. E. Cheng, "A best online algorithm for unbounded parallel-batch scheduling with restarts to minimize makespan," Journal of Scheduling, vol. 14, no. 4, pp. 361-369, 2011.

[6] R. Fu, J. Tian, J. Yuan, and C. He, "On-line scheduling on a batch machine to minimize makespan with limited restarts," Operations Research Letters, vol. 36, no. 2, pp. 255-258, 2008.

[7] R. Uzsoy, C. Y. Lee, and L. A. Martin-Vega, "A review of production planning and scheduling models in the semiconductor industry, part I: system characteristics, performance evaluation and production planning," IIE Transaction on Scheduling and Logistics, vol. 26, no. 5, pp. 47-61, 1994.

[8] R. Uzsoy, C.-Y. Lee, and L. A. Martin-Vega, "Review of production planning and scheduling models in the semiconductor industry part II: shop-floor control," IIE Transaction on Scheduling and Logistics, vol. 26, no. 5, pp. 44-55, 1994.

[9] X. Deng, C. K. Poon, and Y. Zhang, "Approximation algorithms in batch processing," Journal of Combinatorial Optimization, vol. 7, no. 3, pp. 247-257, 2003.

[10] G. Zhang, X. Cai, and C. K. Wong, "On-line algorithms for minimizing makespan on batch processing machines," Naval Research Logistics, vol. 48, no. 3, pp. 241-258, 2001.

[11] C. K. Poon and W. Yu, "On-line scheduling algorithms for a batch machine with finite capacity," Journal of Combinatorial Optimization, vol. 9, no. 2, pp. 167-186, 2005.

[12] P. Liu, X. Lu, and Y. Fang, "A best possible deterministic online algorithm for minimizing makespan on parallel batch machines," Journal of Scheduling, vol. 15, no. 1, pp. 77-81, 2012.

[13] J. Tian, T. C. Cheng, C. T. Ng, and J. Yuan, "Online scheduling on unbounded parallel-batch machines to minimize the makespan," Information Processing Letters, vol. 109, no. 21-22, pp. 1211-1215, 2009.

[14] J. Tian, R. Y. Fu, and J. J. Yuan, “On-line scheduling with delivery time on a single batch machine," Theoretical Computer Science, vol. 374, no. 1-3, pp. 49-57, 2007.

[15] J. J. Yuan, S. Li, J. Tian, and R. Y. Fu, "A best on-line algorithm for the single machine parallel-batch scheduling with restricted delivery times," Journal of Combinatorial Optimization, vol. 17, no. 2, pp. 206-213, 2009.

[16] Y. Fang, P. H. Liu, and X. W. Lu, "Optimal on-line algorithms for one batch machine with grouped processing times," Journal of Combinatorial Optimization, vol. 22, no. 4, pp. 509-516, 2011.

[17] J. Tian, T. C. Cheng, C. T. Ng, and J. Yuan, "An improved on-line algorithm for single parallel-batch machine scheduling with delivery times," Discrete Applied Mathematics, vol. 160, no. 7-8, pp. 1191-1210, 2012.

[18] P. Liu and X. Lu, "Online unbounded batch scheduling on parallel machines with delivery times," Journal of Combinatorial Optimization, 2014.

[19] R. Y. Fu, T. Ji, J. J. Yuan, and Y. X. Lin, “Online scheduling in a parallel batch processing system to minimize makespan using 
restarts," Theoretical Computer Science, vol. 374, no. 1-3, pp. 196202, 2007.

[20] R. Fu, T. C. Cheng, C. T. Ng, and J. Yuan, "Online scheduling on two parallel-batching machines with limited restarts to minimize the makespan," Information Processing Letters, vol. 110, no. 11, pp. 444-450, 2010.

[21] H. Liu and J. Yuan, "Online scheduling of equal length jobs on a bounded parallel batch machine with restart or limited restart," Theoretical Computer Science, vol. 543, pp. 24-36, 2014. 


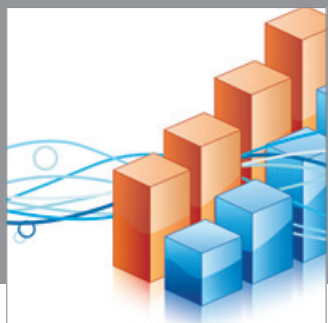

Advances in

Operations Research

mansans

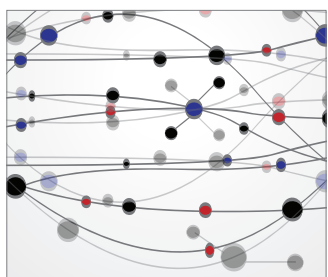

The Scientific World Journal
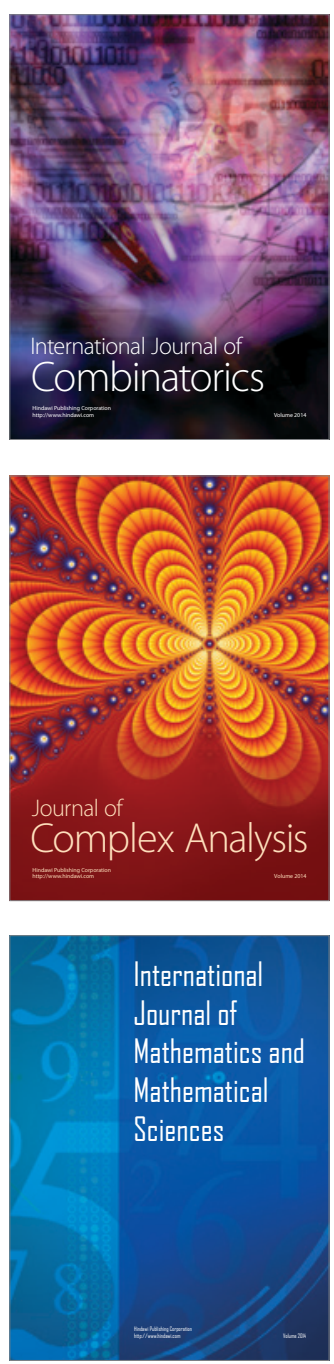
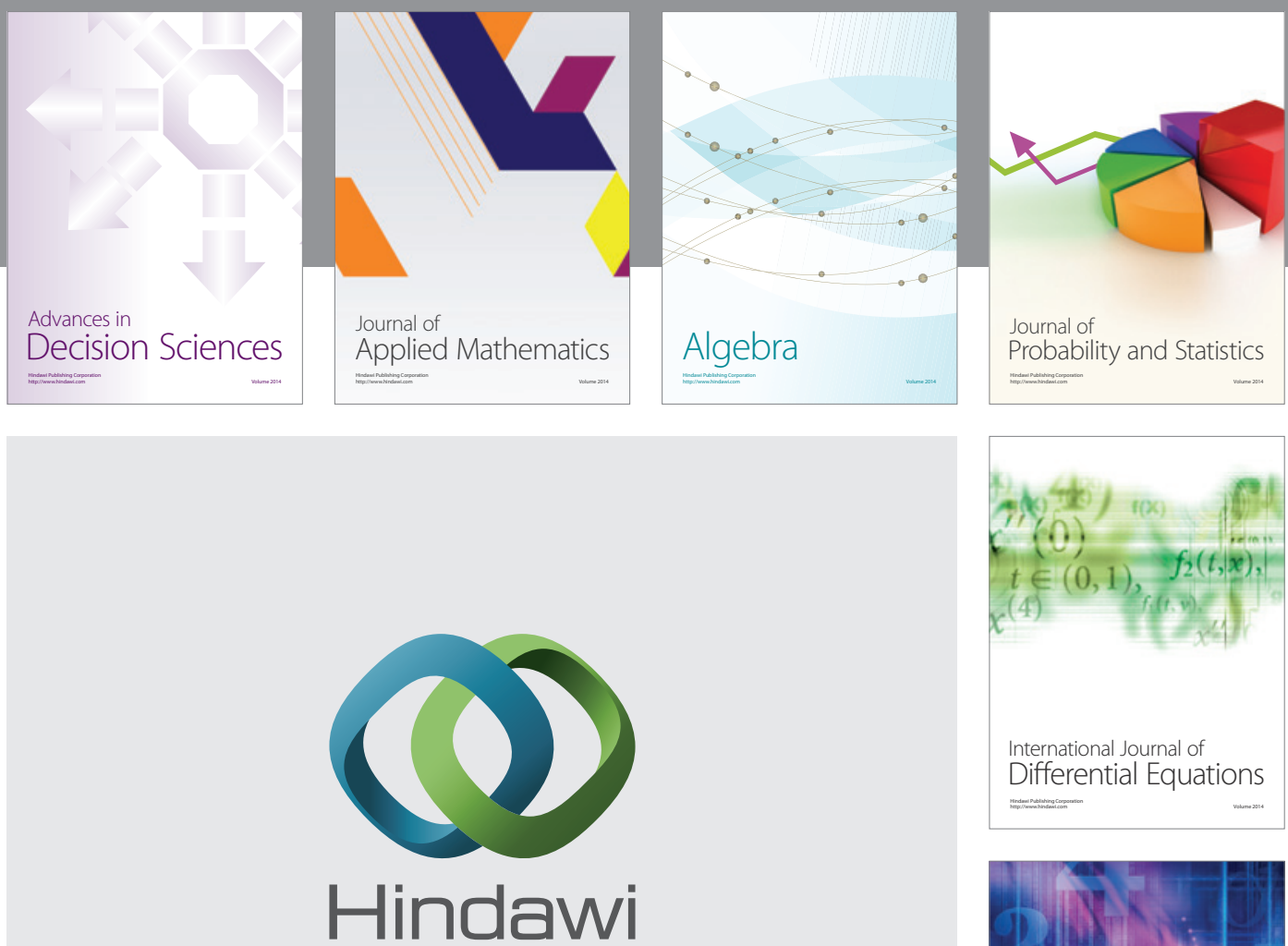

Submit your manuscripts at http://www.hindawi.com
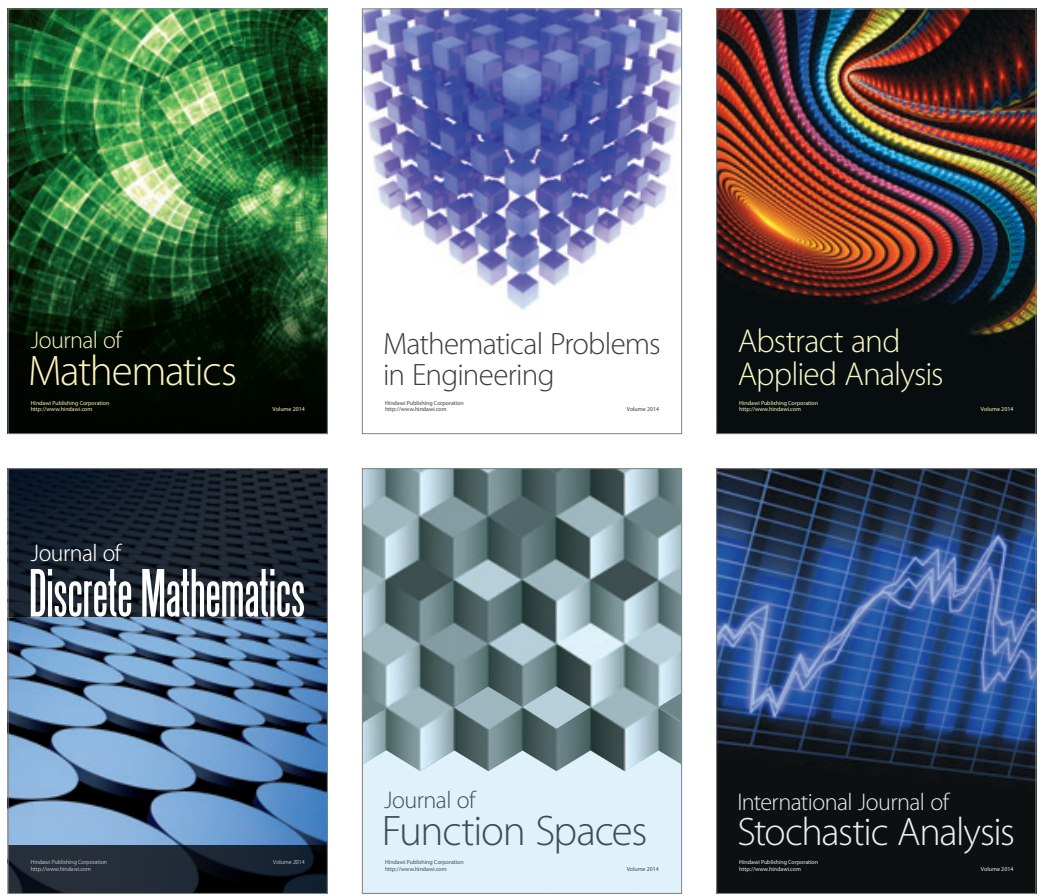

Journal of

Function Spaces

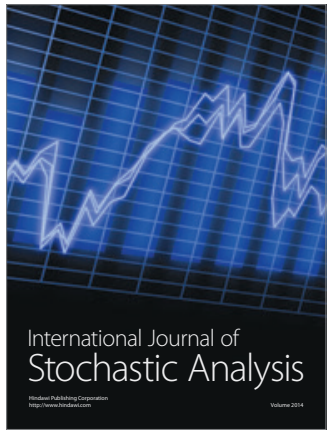

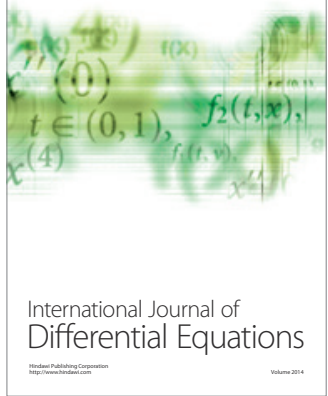
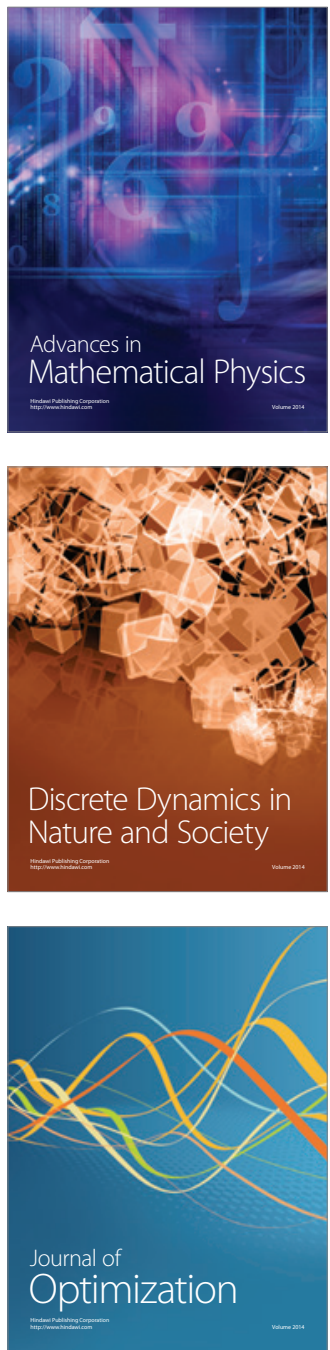